\title{
Denial or decay? The effects of an oral health educational program on caregivers of preschool children
}

\author{
Audrey P. Miller, Chanadra Young-Whiting, Michelle Kameka \\ College of Nursing and Health Sciences, Florida International University, Miami, Florida, USA.
}

Correspondence: Audrey P. Miller. Address: College of Nursing and Health Sciences, Florida International University, Miami, Florida, USA. Email: apemille@fiu.edu

Received: February 28, 2013

Accepted: J une 19, 2013

Online Published: August 21, 2013

DOI : $10.5430 /$ jnep.v4n1p108

URL: http://dx.doi.org/10.5430/jnep.v4n1p108

\section{Abstract}

Background: Dental caries (tooth decay or cavities) are perhaps the most prevalent infectious disease impacting our nation's children. Dental caries in children are five times more common than asthma and seven times more common than hay fever. More than $40 \%$ of children have tooth decay by the time they reach kindergarten. The aim of this study was to examine the effects of an oral health educational program on knowledge and behavior-specific cognitions and affect in caregivers of children ages two to five.

Hypotheses: The five hypotheses determined if there was a positive relationship between: (1) prior related behaviors and behavior-specific cognitions and affect, (2) personal factors and behavior-specific cognitions and affect, (3) preknowledge and prior related behaviors, (4) post-knowledge and intent to provide oral health care, and (5) the difference between caregivers' pre-intervention and post-intervention scores on knowledge.

Methods: Descriptive quasi-experimental study utilizing a pre-test post-test model, with a convenience sample of 400 caregivers recruited from seven Head Start program sites in South Florida, who attended one of 18 oral health educational programs. Research questions addressed the relationship between the oral health educational program and the dependent variables prior related behavior, personal factors, behavior-specific cognitions and affect, knowledge, and intent. Caregivers completed a demographic survey and an oral health behavior questionnaire, a knowledge pre-test, then viewed a 16-minute video designed by Colgate, and completed another knowledge post-test.

Results: The educational program had an overall significant effect on caregivers' prior related behaviors, $r=.43, p$ (two-tailed) $<.01$; behavior-specific cognitions and affect, $r=.43, p$ (two-tailed) $<.01$; intention to provide oral health care for their children, $r=.27, p$ (two-tailed) <.01; and post-test for knowledge of oral health care $(M=60.57, S E=.30)$ compared to pre-test for knowledge $(M=59.03, S E=.26), t(399)=-6.35, p<.01, r=.30)$. However, only $0.4 \%\left(R^{2}=\right.$ $\left..004, \operatorname{Adj} R^{2}=.00\right)$ of the variance in the outcome variable was accounted for by the predictor variables; the model was not significant, $F(2,397)=.80, p=.49$ in comparing personal factors and behavior-specific cognitions and affect.

Conclusions: Poor oral health can compromise a child's overall health and impact their growth and development. Therefore, effective educational programs should be initiated early on to combat widespread oral disease. Effective educational interventions will contribute to positive behavior change and increased knowledge in caregivers. Through health/public policy change, future research will promote preventive oral health care and decrease oral disease.

\section{Key words}

Oral health, Dental caries, Children 


\section{I ntroduction}

The Centers for Disease Control and Prevention ${ }^{[1]}$ reported dental caries (tooth decay or cavities) is perhaps the most prevalent infectious disease impacting our nation's children. Dental caries in children is five times more common than asthma and seven times more common than hay fever. More than $40 \%$ of children have tooth decay by the time they reach kindergarten ${ }^{[2]}$. Infants from families of low socioeconomic status, whose mothers have low education level and who consume sugary foods, are 32 times more likely to have caries at the age of three than children in whom those risk factors are not present ${ }^{[2]}$.

\section{Background}

Oral health care is the primary preventive method of tooth decay (caries or cavities) and infection in children below the age of five ${ }^{[3]}$. Nonetheless, many children still suffer with multiple infectious tooth decay, unnecessary sedative extractions, and tooth loss, resulting in pain, nutritional concerns, and speech delays. Compounding the problem for this population is the possibility of emotional problems due to poor appearance and low self-esteem. Oral health allows individuals to speak, eat, and socialize without disease, discomfort, or embarrassment ${ }^{[4]}$. Oral health is fundamental to general health and well-being and significantly impacts quality of life. Poor oral health can have a detrimental effect on a child's performance in school and success later in life.

Early Childhood Caries (ECC) is a serious disease affecting young children and toddlers, especially those from immigrant ethnic minorities ${ }^{[5]}$. ECC affects the primary teeth of infants and children between the ages of one to six. It can cause cysts, infections, carious eruptions, and malocclusion with the first tooth sighting. A child can have difficulty with speech when the primary teeth are not formed well or lost to extraction due to decay. Furthermore, children are unable to enunciate words or speak clearly if the eruptions of baby teeth are maloccluded (an undesirable positioning of the upper and lower teeth when the jaw is closed). Additionally, nutritional concerns arise when the child is unable to masticate (chew) or swallow food, refusing certain foods that are difficult to chew or have a high content of acid or salt. Moreover, the overall health of the child could be affected with infection and possible systemic sepsis due to infectious eruptions of baby teeth (primary dentition). While the exact cause of ECC is unknown, some factors that are attributed to the disease include nutritional status, transfer of infectious organisms from mother to child, and family size, according to Healthy People $2010^{[6]}$. To prevent this ECC epidemic, Harrison recommended that oral health care behaviors should be established as early as the post-natal period and continue until there is an established dental home ${ }^{[5]}$. Additionally, the researchers declared that the primary prevention of ECC is the establishment of a dental home with routine check-ups and preventive care $^{[7]}$.

The $\mathrm{CDC}^{[1]}$ asserted that community water fluoridation is an effective, safe, and inexpensive way to prevent tooth decay. Community water fluoridation is the addition of fluoride to adjust the natural fluoride concentration of a community's water supply to the level recommended for optimal dental health, approximately $0.7-1.2 \mathrm{mg} /$ liter. New guidelines set forth by Health and Humans Services (HHS) and the Environmental Protection Agency (EPA) to lower the required fluoridation of water to $0.7 \mathrm{ppm}^{[8]}$. In Florida, the current concentration of fluoridated water is $0.8 \mathrm{mg} /$ liter. Specific guidelines and precautions for fluoride use should be taken for children less than six years old and those children at high risk for dental caries $^{[7]}$.

Fluoridation benefits Americans of all ages and socioeconomic status. Fluoride should be given both systemically and topically, whether by fluoridated water in the community or via supplement. However, caution should be used and recommendations should be obtained from the oral health care provider and administered within the needs of the individual child. The American Academy of Pediatrics also recommended optimal exposure to topical and systemic fluoride.

Furthermore, the application of fluoride on children under the age of two should be monitored by a dental professional. According to the CDC and the American Academy of Pediatrics, children under age six years may develop enamel fluorosis if they ingest more fluoride than needed. Enamel fluorosis is a chalk-like discoloration (white spots) on the tooth 
enamel. Therefore, strict observations by the dental professional and family is required for this at risk group of young children.

Like many states, Florida participates in the Head Start (HS) program, a federally funded social program under the umbrella of Community Action Agency for low-income children (age two to five) and their families. There are approximately 1,000,000 children enrolled in HS throughout the country, with 6,500 of those children in South Florida ${ }^{[9]}$. These families must be at or below the current year's poverty guidelines or have a child who has a diagnosed disability to meet program eligibility. This social program offers many services through their perspective unit, e.g., Health, Nutrition, Disability, Mental Health, and Social Services. However, in 2011, Miami-Dade County Governor, Carlos Gimenez, merged several social service departments and created Community Action and Human Services, which now governs HS by clerks and no longer by nurses.

In the Health Unit, there are registered nurses (RN) assigned to South Florida's Head Start (SFHS) centers that perform quality assurance surveillance over the health records of enrolled children. The quality assurance registered nurse (QARN) is responsible for making sure that the health records for the HS children are up to date and complete in order to attend school. There are several health requirements and recommendations for school entrance. The required health documenttation includes a physical exam, immunization record, and PPD. Among the recommended health documentation is laboratory results and an annual dental exam with cleaning, fluoride, and prophylaxis treatment. However, there have been many barriers to obtaining an annual dental exam record, including limited access to pediatric dental providers (pedodontists), lack of dental health insurance, and familial ignorance. Consequently, the QARNs struggle with families and providers to assure and achieve timely oral health care and treatment for these children. In California, for example, $85 \%$ of the children in their HS program received dental exams; of those, $29 \%$ needed treatment, and $84 \%$ received follow-up care. However, in SFHS programs, only $78 \%$ of the children received dental exams, $17 \%$ of those needed treatment, and $86 \%$ received follow-up care ${ }^{[9]}$.

Florida Medicaid encompasses preventive dental coverage for children 20 years and younger. This coverage includes a dental exam every six months that provides cleaning, examination, and prophylaxis treatment. In some cases, the coverage will include extractions, restorations, and sealants ${ }^{[10]}$. At the time of this study, Atlantic Dental Incorporated (ADI), now known as DentaQuest, was the Health Maintenance Organization (HMO) for dental care and coverage for Miami-Dade County. ADI utilized local dental providers and governed where recipients would receive services. Now there is a Prepaid Dental Health Plan (PDHP) that is an additional Medicaid managed dental care option in Miami-Dade County ${ }^{[10]}$.

\section{Statement of the problem}

The problem is that not all two to five year-old children in South Florida receive routine preventive oral health care; therefore, they have poor oral health. Familial involvement is oftentimes minimal and non-compliant when it comes to taking children to the dentist for oral health care. Therefore, the assumptions were made that caregivers and/or families of this group of children lacked knowledge in oral health care, lacked understanding in the importance of oral health care, and lacked the tools necessary to provide preventive oral health care for their children. As a result, these children did not receive the appropriate anticipatory guidance for proper oral health care to maintain healthy teeth and gums and are at risk for oral disease.

\section{Purpose}

The purpose of this investigation was to examine the effects of an oral health educational intervention on prior related behavior, personal factors, behavior-specific cognitions and affect (perceived benefits, perceived barriers, and perceived self-efficacy), knowledge, and intent in caregivers of children between the ages of two and five.

\section{Theoretical framework}

This study was designed to examine the effects of an oral health educational intervention on prior related behavior, personal factors, behavior-specific cognitions and affect (perceived benefits, perceived barriers, and perceived self- 
efficacy), knowledge, and intent in caregivers of children between the ages of two and five. The theoretical framework for this study was based on the Health Promotion Model (HPM) that explored the factors and relationships contributing to health-promoting behavior and therefore to the enhancement of health and quality of life ${ }^{[10]}$. For the purposes of this study, this researcher utilized a modified version of the three major concepts in the HPM: (1) individual characteristics and experiences, (2) behavior-specific cognitions and affect, and (3) behavioral outcomes ${ }^{[11]}$. Individual characteristics and experiences demonstrate that each caregiver has unique personal characteristics and experiences that affect subsequent actions. Behavior-specific cognitions and affect variables within the HPM are considered to have major motivational significance. Measuring change in these variables is essential to determine if such changes actually result from the oral health intervention and, in turn, influence changes in commitment or in the occurrence of health-promoting behaviors. Behavioral outcomes included oral health-promoting behavior and intent is the end point or action outcome in the HPM. These behaviors, when integrated into a healthy lifestyle that permeates all aspects of living, result in improved oral health, enhanced functional ability, and better quality of life at all stages of development ${ }^{[1]}$. See Figure 1 for revised model.

\section{Significance of the problem}

The significance of this selected problem was that decay of primary teeth can affect children's growth, lead to malocclusion, and result in significant pain and potentially life-threatening swelling ${ }^{[7]}$.

\section{Hypothesis}

Among caregivers for children between the ages of two and five, there is a positive predictive relationship between caregivers' personal factors of age and income and their behavior-specific cognitions and affect (perceived benefits, perceived barriers, and perceived self-efficacy) related to providing oral health care for their children. For the purposes of this manuscript, only hypothesis 2 will be discussed.

Figure 1. Miller (2011a).

Adapted version, modified with researchers'

permission, from Pender's Health Promotion Model, related to oral health care. Permission to reprint.

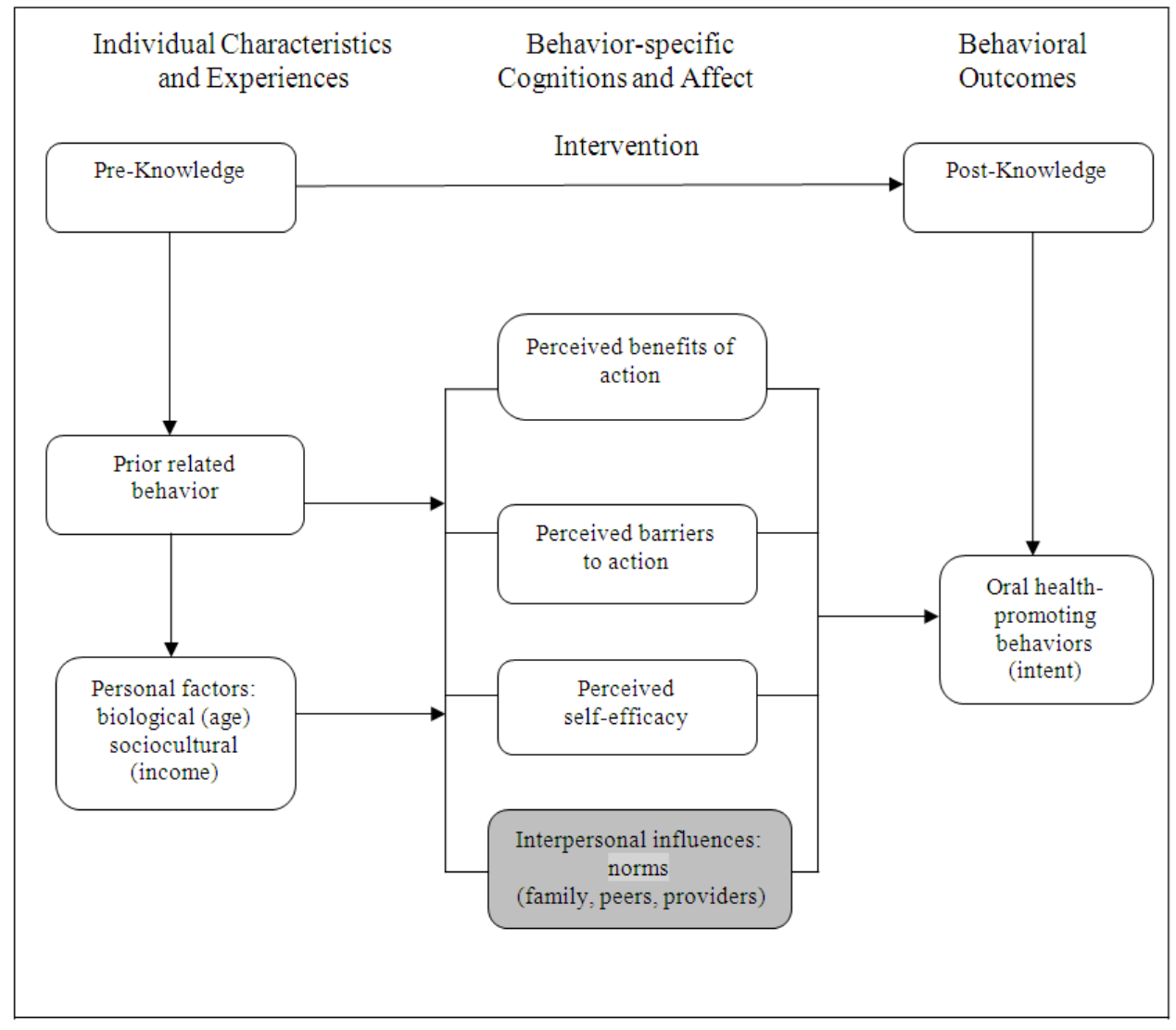




\section{Methods}

\section{Design}

This was a descriptive quasi-experimental pre-test post-test design, with a convenience sample. The researcher presented an oral health educational program at the SFHS sites for caregivers who chose to participate. Determinants of Oral Health Behaviors (DOHB), personal factors instrument, and the pre-Oral Health Knowledge Test (OHKT) was given initially. After the educational intervention, the post-OHKT and intent questionnaire was administered.

\section{Sample}

A convenience sample of 400 caregivers of children who were enrolled in the SFHS program were recruited from attendees at 18 oral educational programs scheduled at seven sites. The criteria for sample selection included providing a balance from the SFHS program. Therefore, the researcher attempted to gather a sample of caregivers from the north, central, and south regions of the county, as there were approximately 6,400 children enrolled in the program. The inclusion criteria were that the participants were caregivers and their children who have qualified for and are enrolled in the SFHS program. The caregivers were English-speaking and the child was between the ages of two and five. The exclusion criteria were anyone not enrolled in the HS program, did not speak English and their child was younger than two years old or older than five years old.

\section{Educational program}

At the start of the educational program session information was provided about the study and the cover letter reviewed. Caregivers in attendance, who agreed to participate, remained in the room. Those caregivers who chose not to participate in the study still partook in the educational program and received the benefits of oral health care knowledge. To maintain confidentiality, the envelope did not have any identifiable marks on the outside. The oral health program was a 16-minute video entitled, "Dr. Rabbit and the Legend of Tooth Kingdom", which was developed by Colgate Bright Smiles, Bright Futures. This video provided basic information on oral health care for Pre-K, Head Start, and families. At the end of the video, there was a 10-minute review by the researcher of the content of the video, followed by a question and answer period. In its entirety, the oral health educational program took approximately 1.5 hours to complete. When the participants exhausted their questions, they completed the post-test (color coded blue), which contained only the OHKT.

\section{I nstruments}

\section{Determinants of oral health behavior}

The Determinants of Oral Health Behaviors (DOHB) instrument was developed by Morowatisharifabad and Shirazi ${ }^{[4]}$ to determine if behavior-specific cognitions and affect would affect oral health behaviors. The development of the instrument combined similar scales from Pender's Revised Health Promotion Model constructs in other areas such as exercise and health-promoting lifestyle using Iranian $12^{\text {th }}$-grade students. A panel of five Iranian experts in the field of health behavior, education, and oral health were asked to evaluate the pilot instrument for appropriateness and relevance of items, reading level, and response format.

The DOHB instruments investigated multiple determinants of oral health, including oral health behaviors, a 13-item scale with an internal consistency coefficient of 0.65 ; perceived self-efficacy for oral health behaviors, a 10-item scale with an internal consistency coefficient of 0.81 ; perceived benefits of actions, a 7-item scale with an alpha coefficient of 0.79; and perceived barriers scale, a 9-item scale with a Cronbach's alpha of 0.76; interpersonal influences (norms), a 5-item scale with an alpha coefficient of 0.65 ; intent for oral health behaviors, a 10-item scale with an internal consistency coefficient of 0.81 . All responses were scored on a Likert-scale format. The activity-related affect scale, interpersonal modeling, and situational influences scale was not utilized in this study ${ }^{[4]}$.

The dependent variables prior related behavior, personal factors, behavior-specific cognitions and affect (perceived benefits, perceived barriers, and perceived self-efficacy), and intent were measured by the Determinants of Oral Health 
Behavior (DOHB) instrument, created by ${ }^{[4]}$. The DOHB (Oral Health Behaviors sub-set) is a 15 -item Likert instrument designed to measure behaviors towards oral health care, with a score of 1 (never), 2 (occasionally), 3 (often), and 4 (always). The higher the number of responses, the more likely there will be health-promoting behaviors towards oral health care. The DOHB (Perceived benefits sub-set) is a 7-item Likert instrument designed to measure benefits of oral health care using 1 (strongly disagree), 2 (disagree), 3 (agree), and 4 (strongly agree). The higher the number of responses, the more perceived benefits to oral health care. The DOHB (Perceived Barriers sub-set) is a 9-item Likert instrument designed to measure obstacles to oral health care, with a score of 1 (never a problem), 2 (occasionally a problem), 3 (oftentimes a problem), and 4 (always a problem). The greater the number of responses, the more perceived barriers to oral health care. The DOHB (Perceived self-efficacy sub-set) is a 15-item Likert instrument designed to measure self-efficacy towards oral health care, with a score of 1 (completely unable), 2 (somewhat unable), 3 (somewhat able), and 4 (completely able). The higher the number of responses, the greater self-efficacy towards oral health care.

\section{Personal factors and intent}

The personal factors instrument was a researcher-developed survey consisting of a 10-item open-ended questionnaire. The questions specifically addressed the caregivers' demographic status. Two questions addressed the caregivers' views on oral health care. This researcher revised intent questionnaire is a 15-item instrument designed to measure the caregiver's plan to provide oral health care for their children post-educational intervention. The scores are 1 (very likely), 2 (unlikely), 3 (likely), 4 (very likely). The higher the score, the greater the prediction, that caregivers provided oral health care to their children after the oral health educational intervention.

As these instruments were not previously used to measure these variables among caregivers of children between the ages of two and five, this researcher calculated Cronbach's alpha to ensure internal consistency with this population. With the permission of the instrument's developer, the instruments were modified by this researcher for appropriate fit for this population. Additionally, while the intent instrument was developed by Dr. Morowatisharifabad, it had never been used previously to measure intent. Measures of reliability were conducted to assure that the intent instrument was internally consistent with this population.

\section{Oral health knowledge test}

Knowledge refers to the individual's ability to obtain, understand, and act upon health information and to make appropriate health decisions ${ }^{[12]}$. Oral health knowledge is the individual's ability to obtain, understand, and make appropriate decisions to maintain healthy oral health behaviors and habits. Caregiver knowledge was measured by the OHKT. The OHKT is a 17-item knowledge and attitude test and a 7-item pictorial test (with 17 possible answers), a subset of the Oral Health Literacy Instrument (OHLI) created by Lawrence, Romanetz, Cappel, Binquis, and Rodgers ${ }^{[13]}$. The 17-item Likert instrument was designed to measure the oral health knowledge and attitudes of caregivers, with a score of 1 (strongly disagree), 2 (disagree), 3 (agree), and 4 (strongly agree). The higher the total score, the more knowledgeable the caregiver is on oral health. In the 7-item pictorial tool, wherein a score of 1 (correct) and 0 (incorrect). The higher the total score, the more knowledgeable the caregiver is on oral health.

\section{Reliability and validity for DOHB instrument}

The DOHB instrument was pilot-tested for reliability and validity in a convenience sample of 30 Iranian pre-university students. Psychometric testing indicated satisfactory internal consistency and validity of the instruments for this group of Iranians adolescents. All the instruments had acceptable reliabilities with alpha coefficients greater than 0.65 . Construct validity was supported by confirmatory factor analysis ${ }^{[4]}$.

\section{Reliability and validity for OHKT instrument}

Internal consistency reliability of the OHLI and the OHKT was determined by using Cronbach's alpha for the overall sample and test-retest reliability using the intra-class correlation coefficient (ICC) for the 20 patients who completed the questionnaire twice. Face validity was established by three experts in the fields of dental public health, preventive dentistry, and health literacy. Content validity was enhanced by incorporating the aforementioned materials and texts often encountered by patients in a dental care setting. Concurrent validity was tested by comparing OHLI scores across cate- 
gories of education level and frequency of visits to health care providers, which are known to vary in medical health literacy. Construct validity was assessed by correlating the total OHLI scores with the scores on the Test of Functional Health Literacy in Adults (TOFHLA) and the test of the OHKT using Spearman's rank correlation. All instruments had acceptable reliabilities with alpha coefficients greater than .65. Construct validity was supported by confirmatory factor analysis. Sabbahi et al. ${ }^{[12]}$ and Schroth, Brothwell, and Moffatt ${ }^{[14]}$ both utilized and successfully tested the OHKT in their studies.

\section{Ethical considerations}

This was a minimal risk study. However, there might have been a risk of fatigue or embarrassment on the part of the caregivers. Therefore, confidentiality was maintained at all times throughout this study. In conducting research with children, the researcher assured others who acted as gatekeepers that risk to the participants remained minimal. The hierarchy of gate keeping to protect the children and their families comprised of the University Institutional Review Board (IRB) and the Executive Committee and designated program administrator from the Head Start program. At any time, the caregiver could have opted out of participating in this study without fear of denial of care or treatment. Additionally, any participant could have refused to participate and opted out at any time during this study without repercussions from agency personnel or denial of services guaranteed by HS.

Nevertheless, caregivers could have continued to have the opportunity to enjoy the educational intervention and small dental gift items distributed at the end of the program session. There was no direct benefit of the study to the family, but education on oral health care received and the oral health care gift packs for the children benefited them all in the long term. These gifts packs included a toothbrush, toothpaste, floss, brushing timer, a pencil and eraser, and a flyer that provided dental tips.

\section{Data collection}

Participants were issued an envelope with two color coded packets for the pre- and post-test. The first packet, which was used for the pre-test, included the OHKT, personal factors questionnaire, and the DOHB. Participants were asked to complete these three questionnaires and to assign a unique identifier on the upper right-hand corner of the forms. The purpose of this unique code was to ensure that the pre-test were properly linked to the post-test to minimize errors. Upon completion of the questionnaires and the pre-test, which was color coded red, the participants were asked to clip them together and return them to the envelope. After completing the tools, the educational intervention began. At the conclusion of the program, the participant's completed the post-test.

\section{Data analysis}

Descriptive statistics were conducted on the total sample for all major study variables, including frequencies and measures of central tendency to describe the sample. Data were analyzed using IBM $^{\circledR} \operatorname{SPSS}^{\circledR}$, version $19.0^{[15]}$. Hypotheses testing were conducted using techniques of $t$-test for dependent means, Pearson's correlation coefficients to examine the relationships between the variables, and multiple regressions to predict intent. The level of significance used in this study was an alpha level 0.05 . Power Analysis was determined by utilizing the $G^{*}$ Power 3.1 program. After careful input into the $\mathrm{G}^{*}$ Power 3.1 calculator $(\alpha=.05,1-\beta=0.8, d=0.5)$ for $t$ - test for dependent variables $(n=27)$ and Pearson's $\mathrm{r}$ correlation $(n=342)$, the total sample size was calculated to be $n=369$. In order to account for errors, missing data, mislabeled, or blank information on any survey, questionnaire, or test, this researcher used a sample size of 400 . While the original research study contained and tested five hypotheses, only the results of hypothesis 2 will be discussed.

\section{Results}

\section{Sample}

The participant sample consisted predominantly of Black women $(n=308,77 \%)$ between the ages of 18 and 67 years of age with Hispanics $(\mathrm{n}=21,5.2 \%)$ the second most prominent group. The majority of caregivers were single $(\mathrm{n}=253$, 
63.2), and only $40.2 \%$ graduated with a high school diploma. All but 31 (7.8\%) had not completed high school or the high school equivalency exam (GED); 95 individuals (23.8\%) reported they had attended a college or university. Employment status was reported with 106 (26.5\%) working on a full-time basis, 133 (33.2\%) working part time, and 161 (40.2\%) being unemployed. The majority $(\mathrm{n}=303,75.8 \%)$ reported that they used Medicaid funds to pay for their child's health care, and another 54 (13.5\%) were enrolled in Florida KidCare; 43 (10.8\%) had private insurance to pay for their child's oral healthcare.

The participants were asked to report how they would describe their child's oral health condition in general. The majority $(\mathrm{n}=257,64.2 \%)$ thought their child's oral health condition was good; $92(23.0 \%)$ described the oral health as excellent, and $51(12.8 \%)$ responded that the child's oral health condition was poor. Then, the participants were asked specifically if they thought their child had any dental disease or cavities. Most $(n=299,74.8 \%)$ thought their child did not have any dental disease or cavities, 90 (22.5\%) responded yes, and 11 (2.8\%) reported that they did not know.

\section{Influences of interpersonal norms}

The participants were asked to indicate how often typical referents encouraged them to provide oral care for the child. Typical referents included their father, mother, relatives, friends and/or a dental provider. The participants reported that their fathers often encouraged oral health care for the children $(\mathrm{n}=135,33 \%)$, mothers always encouraged oral health care $(n=227,56 \%)$, relatives occasionally encouraged oral health care $(n=140,35 \%)$, friends occasionally encouraged oral health care $(n=196,51 \%)$, and their dental provider always encouraged oral health care $(n=329,82 \%)$.

\section{Relationship between personal factors and behavior-specific cognitions and affect}

Among caregivers for children between the ages of two and five, there is a positive predictive relationship between caregivers' personal factors of age and income and their behavior-specific cognitions and affect related to providing oral health care for the children (benefits, barriers, and self-efficacy). The research hypothesis was rejected. Regression analysis found no significant relationship between the predictor variables of age and income to the outcome variable of behaviorspecific cognitions and affect related to providing oral health care for the children. Only $0.4 \%\left(R^{2}=.004\right.$, Adj $\left.R^{2}=.00\right)$ of the variance in the outcome variable was accounted for by the predictor variables; the model was not significant, $F(2,397)$ $=.80, p=.49$. Please see Table 1 for a full review of analysis and results.

\section{Discussion}

Statistical analysis revealed that Hypothesis 2 was rejected, while hypotheses 1, 3, 4, and 5 were accepted. The results for Hypothesis 2 did not support a significant relationship between personal factors (age and income) and behavior-specific cognitions and affect (perceived benefits, perceived, barriers, and perceived self-efficacy). Hypothesis 1 was supported, indicating a significant relationship between prior related behavior and behavior-specific cognitions and affect (perceived benefits, perceived barriers, and perceived self-efficacy). Hypothesis 3 was supported, indicating a significant relationship between pre-knowledge and prior related behaviors. Hypothesis 4 was supported, indicating a significant relationship between, post-knowledge and caregiver's intention to provide oral health care for their children. Hypothesis 5 was supported, indicating that caregivers scored significantly higher on post-knowledge test than pre-knowledge test on oral health care for their children.

Findings from hypotheses 2 were supported by Adair, Pine, Burnside, Nicoll, Gillett, \& Anwar et al. ${ }^{[16]}$. They researched familial and cultural perceptions and beliefs of oral hygiene and dietary practices ethnically diverse groups. The aim of this international study was to develop a valid and reliable psychometric measure to examine the extent to which caregivers' attitudes about engaging in twice-daily toothbrushing and controlling sugar snacking would predict these individual behaviors in children. A supplementary objective was to assess whether ethnic group, culture, level of deprivation, or children's caries experience impact upon the relationship between oral health-related behaviors, attitudes to these respective behaviors and to dental caries. 
Table 1. Quantitative Analysis Results Grid

\begin{tabular}{l}
\hline Research Question \\
1. Among caregivers for \\
children between the ages \\
of two and five, do the \\
caregivers' behavior of \\
providing oral hygiene for \\
the children prior to an \\
educational intervention \\
correlate with their \\
behavior-specific \\
cognitions and affect related \\
to providing such care \\
(benefits, barriers, and \\
self-efficacy)?
\end{tabular}

\section{Among caregivers for} children between the ages of two and five, do the caregivers' personal factors of age and income correlate with their behavior-specific cognitions and affect related to providing oral hygiene care for the children (benefits, barriers, and self-efficacy)?

\section{Among caregivers for} children between the ages of two and five, is there a positive correlation between caregivers' knowledge of oral hygiene prior to an educational intervention and their behavior of providing oral hygiene to the children prior to the intervention?

\section{Among caregivers for} children between the ages of two and five, is there a positive correlation between caregivers' postintervention knowledge of oral hygiene and their intention to provide oral hygiene to the children following the intervention?

5. Is an educational intervention effective in increasing the knowledge regarding providing oral hygiene care to children for a group of caregivers of children between the ages of two and five?

Hypotheses
1. Among caregivers of
children between the ages of
two and five, there is a
positive correlation between
the caregivers' behavior of
providing oral hygiene for the
children prior to an
educational intervention and
their behavior-specific
cognitions and affect related
to providing such care
(benefits, barriers, and
self-efficacy).

\section{Among caregivers for} children between the ages of two and five, there is a positive predictive relationship between caregivers' personal factors of age and income and their behavior-specific cognitions and affect related to providing oral hygiene care for the children (benefits, barriers, and self-efficacy).

3. Among caregivers for children between the ages of two and five, there is a positive correlation between caregivers' knowledge of oral hygiene prior to an educational intervention and their behavior of providing oral hygiene to the children prior to the intervention.

4. Among caregivers for children between the ages of two and five, there is a positive correlation between post-intervention scores for knowledge of oral hygiene and their intention to provide oral hygiene to the children.

$\begin{array}{lll}\text { Instrument } & \text { Statistical Test } & \text { Results } \\ \text { Determinants of } & \text { Pearson's } r & r=.43, p<.01 \\ \text { Oral Health } & \text { simple } & \text { (two-tailed). Significant } \\ \text { Behaviors } & \text { correlation, } & \text { relationship between } \\ \text { (DOHB) } & p<.05 & \begin{array}{l}\text { scores for prior behavior } \\ \text { and the behavior-specific } \\ \text { 46 items }\end{array} \\ & & \text { cognitions and affect. } \\ & \text { Effect size was medium. }\end{array}$

DOHB and Pearson's $r$ Demographic Survey (DS) 56 items

\section{multiple} correlation, $p<.05$

\section{(Multiple} regression)

DOHB, DS, and

Oral Health

Knowledge Test

(OHKT)

80 items

Pearson's r simple correlation, $p<.05$

$\begin{array}{lll}\begin{array}{l}\text { OHKT } \\ \text { Intent } \\ 39 \text { items }\end{array} & \text { and } & \begin{array}{l}\text { Pearson's } r \\ \text { simple } \\ \text { correlation, } \\ \end{array} \\ & & <.05\end{array}$

\section{There is a significant} difference between the pre-intervention scores and the post-intervention scores for knowledge of oral hygiene among a group of caregivers of children between the ages of two and five.

\section{OHKT (pre) and $t$ - test for OHKT (post) dependent 48 items variables, $p<.05$}

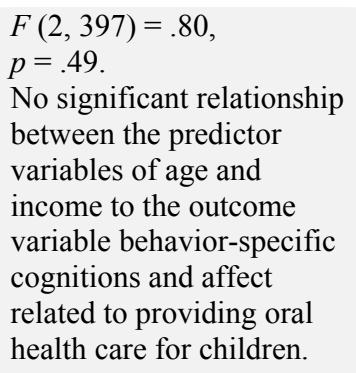
between the predictor variables of age and income to the outcome variable behavior-specific cognitions and affect related to providing oral health care for children.

\section{$r=.16, p<.01$ (two-tailed). Significant relationship between the scores for caregivers' knowledge prior to the educational intervention and their prior related behaviors. Effect size was small.}
$r=.27, p<.01$ (two-tailed). Significant relationship between the post-intervention scores for knowledge and caregivers' intent to provide oral health care for their children. Effect size was small.
$(M=60.57, S E=.30),(M$ $=59.03$, $S E=.26), \mathrm{t}(399)-6.35, p<$ $.01, r=.30)$. Caregivers scored significantly higher on post-test for knowledge than they did for pre-test knowledge. Effect size was small.


The results indicated that factor analysis identified eight coherent attitudes towards toothbrushing, sugar snacking and childhood caries. Attitudes were significantly different in families from deprived and non-deprived backgrounds and in families of children with and without caries. Caregivers' perception of their ability to control their children's toothbrushing and sugar snacking habits were the most significant predictors of whether or not favorable habits were reported. In conclusion, this study supports the hypothesis that caregiver attitudes significantly impact on the establishment of habits favorable to oral health ${ }^{[16]}$.

Adair et al. study concluded that attitudes toward oral health care significantly differed among families socioeconomical backgrounds ${ }^{[16]}$. This research also showed that there was no correlation or significant relationship between age and income in relation to behavior-specific cognitions and affects (perceived benefits, perceived barriers, and perceived self-efficacy). Therefore, no matter the age or annual income of the household, there was no impact on the caregivers' perceived attitudes or practices towards oral health care for their children.

There is a gap in knowledge as it relates to children two to five years old and the effects that preventive oral health care can contribute to a healthy lifestyle. Preventive care is minimized by the lack of knowledge by caregivers. The children of this population go without anticipatory guidance, preventive care, and/or restorative care. By the time, oral health providers examine these children, tooth decay and damage is rampant. Care for the school-age child is more readily available, as parents will care for the permanent dentin (teeth) before the primary dentin.

Determining the caregivers' knowledge and behavior-specific cognitions and affect (perceived benefits, perceived barriers, and perceived self-efficacy) could inform health care providers about necessary steps to educate the public on the promotion and outcomes of early oral health care for children. Most participants were eager to learn about preventive oral health care for their children. They were surprised that simple oral health care at home would prevent early childhood caries. There are still parents who need reinforcement on the importance of oral health care for these children. However, this oral health educational intervention significantly and positively increased these caregivers' knowledge and promoted oral health behaviors and intent for their children between the ages of two and five.

At the time of this study, it is unknown whether the children of the HS caregiver's received prior dental care or had access to a pediatric dental provider (pedodontists) for oral health care. Although, fluoride varnish treatments are not recommended for children less than eight years old (unless they are at high risk for tooth decay, have no fluoridated water system, and are under strict supervision of a dental provider), it is unknown if the children of this particular population received fluoride varnish treatments before or after the conclusion of this study.

\section{Significance of the study}

The significance of this study was determining that caregivers' knowledge and behavior-specific cognitions and affect (perceived benefits, perceived barriers, and perceived self-efficacy) could inform health care providers about necessary steps to educate the public on the promotion and outcomes of early oral health care for children. The oral health educational intervention significantly and positively increased caregivers' knowledge and promoted oral health behaviors and intent for their children between the ages of two and five. However, socioeconomic status and age had no determining factor of caregiver's knowledge and attitudes towards oral health care for their children.

\section{Nursing education}

Education is the key to increasing preventive care, thereby reducing health complications. Furthermore, this investigation identified the relationship between familial attitude and early oral health care for children. After the educational intervenetion, the caregivers' understood the importance of early preventive oral health care for their children, as shown in the significantly higher post-test scores for knowledge and intent to provide oral health care. Emphasizing oral health care for all populations in the nursing curriculum, particularly in children, will contribute to greater adherence to oral health practices by caregivers and other segments of the population who are currently uninformed. This research showed that the 
caregivers' lack of oral health knowledge affected the way they provided oral health care for their children, which in turn, caused a lack in perceived action towards oral health. Formal educational interventions designed for venues like school health and public health centers are other programs that should be implemented.

\section{Nursing practice}

Nurses have the responsibility to make sure their patients' dental care needs have been met and that the risks of oral disease and potential systemic infection are reduced ${ }^{[17]}$. The implications for nursing practice are to implement educational programs for caregivers that would lead to the reduction the incidences of children's tooth decay, tooth loss and pain, thereby reducing speech delays, nutritional complications, and increasing overall growth and development. The outcomes of an oral health care educational intervention and promotion program, led by nurses, school nurses, and nurse researchers, will ultimately increase preventive oral health care and decrease symptoms and disease ${ }^{[18]}$. Demonstrating that health promotion and disease prevention strategies have favorable outcomes for the community is significant to nurses and nursing science.

\section{Nursing research}

Nurse researchers increase knowledge to the discipline. Increased knowledge not only benefits the discipline but also benefits the patient and the community. Nurse researchers are often the health care providers who test and evaluate health promotion interventions and are knowledgeable about services available to at-risk children. This study revealed a signifycant increase in knowledge after the oral health educational intervention. This study also addressed an increase in caregivers' awareness for the need of oral health education, oral health-promoting behaviors, and the intent to provide oral health care for their children.

The data obtained in this research study provided results that support nurses with the ability to initiate collaborative efforts with other health care agencies, the potential to commence grant proposals for oral health care programs and services, and the opportunity to affect a change in governmental policy (through evidenced-based practice and interventions) that will ultimately support the continued need for oral health care services for young children.

\section{Health/ public policy}

Healthy People $2020^{[20]}$ initiative, now in its third decade, is a health initiative that provides science-based or evidencedbased recommendations to improve the health of all Americans. A few goals of the initiative are to attain longer lives free of preventable disease, to eliminate disparities and improve health, and to promote healthy development and healthy behaviors. The objectives from Healthy People 2020 suggests the reduction in dental caries (tooth decay) in primary teeth and the amount of untreated dental caries (tooth decay) in children between the ages of three and five from low-income families.

Therefore, accessible free or low cost oral health care clinics should be available to all children under the age of 18 to ensure adequate services that will prevent oral disease and promote oral health. Mandatory oral health exam for all children entering school should be enforced, like immunizations and physical exams, to maintain the total healthy child. There should be an increase in the amount of Federally Qualified Health Centers throughout South Florida with an oral health component, oral health educational program, and oral health services that will provide care to children from low-income families.

The nursing implications dictate that education is the key to increasing preventive care, thereby reducing health complications. Furthermore, this investigation identified the relationship between familial attitude and early oral health care for children. After the educational intervention, the caregivers' understood the importance of early preventive oral health care for their children, as shown in the significantly higher post-test scores for knowledge and intent to provide oral health care. Emphasizing oral health care for all populations in the nursing curriculum, particularly in children, will contribute to greater adherence to oral health practices by caregivers and other segments of the population who are 
currently uninformed. This research showed that the caregivers' lack of oral health knowledge affected the way they provided oral health care for their children, which in turn, caused a lack in perceived action towards oral health. Oral health educational interventions for caregivers are necessary in early childhood programs. Not only to increase the caregivers' knowledge base, but will be an effective method in disease prevention and oral health care promotion. Formal educational interventions designed for venues like school health and public health centers are other programs that should be implemented despite caregiver age and/or socioeconomic status.

\section{Limitations of the study}

The utilization and benefits of an oral health educational intervention was significant in increasing caregiver knowledge and intent to provide oral health care for their children. However, the following limitations to this study are as follows: At the time of data collection, the researcher was present, and this might have caused the caregivers to answer with socially acceptable responses; the sample was drawn from a social program designed to enroll families with low income; convenience sampling may have been biased, thereby limiting the generalizability of the study findings; there was poor caregiver participation and staff involvement at HS sites with student enrollment greater than 140; even with advertising and supportive recruitment efforts by the staff at smaller HS sites, many caregivers were either not interested, did not have the time, avoided the educational intervention all together, or requested more incentive for study participation; the oral health care educational intervention took caregivers approximately 90 minutes to complete causing some participants to answer questions without thought; self-reported answers by the caregivers may have been based on social desirability rather than actual belief and practice; therefore, inaccurate answers may have been given. Although the Head Start sample size for this study was composed of $77 \%$ Black participants, the most limiting issue for this research study was the inclusion criteria of English-speaking persons only in a Metropolitan county that is predominantly Hispanic.

\section{Conclusion}

The findings from this study will influence other quantitative studies to explore additional variables and populations that might persuade the need for an effective oral health educational intervention that will increase health-promoting behaviors and intent in caregivers and in children. Additional post-test opportunities after the intervention and a longitudinal study are needed in future research to determine if knowledge was retained and intent quantified. As well as inclusion of Spanish-speaking Head Start participants utilizing measuring tools accurately translated into Spanish for a more generalizable outcome in South Florida. While this study was a quantitative analysis of an underserved, underprivileged population of caregivers, a qualitative research study might have revealed why these caregivers do not take their children for preventive oral health care or what barriers were instrumental in preventing them from accessing oral health care services. The Health Promotion Model was a significant foundation for this study to utilize educational promotion techniques and guidance for health promotion.

It is hoped by this researcher that caregivers will increase the knowledge and oral health-promoting behaviors to provide oral health for their children, thereby decreasing oral disease. Additionally, knowledge retention and intention to provide oral health care for their children, will improve oral health outcomes for children. The goal is to change public policy, increase financial support for oral health care services for children, and increase access to oral health care for children from low-income families, thereby decreasing oral health disease through oral health prevention and promotion.

\section{References}

[1] CDC. (Centers for Disease Control and Prevention). Oral Health 2000: Facts and figures. [updated 2013 Jul 13; cited 2010 Aug]. Available from: http://www.cdc.gov/OralHealth/publications/factsheets/

[2] Allukian M. The neglected epidemic and the surgeon general's report: A call to action for better oral health. Amer J of Pub Hlth. 2000; 90(6): 843-45. http://dx.doi.org/10.2105/AJPH.90.6.843

[3] DHHS. (Department of Health and Human Services). Oral health in America: A report of the surgeon general. MD: DHHS, National Institutes of Health, National Institute of Dental and Craniofacial Research; 2000b. 13. 
[4] Morowatisharifabad MA, Shirazi KK. Determinants of oral health behaviors among preuniversity (12th-grade) students in Yazd (Iran). Fam Comm Hlth. 2007; 30(4): 342-50. PMid:17873640http://dx.doi.org/10.1097/01.FCH.0000290546.33328.76

[5] Harrison RL, Wong T. An oral health promotion program for urban minority population of preschool children. Comm Den and Oral Epi. 2003; 31: 392-99. http://dx.doi.org/10.1034/j.1600-0528.2003.00001.x

[6] DHHS. (Department of Health and Human Services). Healthy People 2010. DC: U.S. Government Printing Services; $2000 \mathrm{a} .43$ p.

[7] Hale KJ, Weiss PA, Czerepak CS, Keels MA, Thomas HF, Webb MD. Oral health risk assessment timing and establishment of the dental home. Am Acad of Ped. 2003; 3(5): 1113-16.

[8] Health and Human Services. HHS and EPA announce new scientific assessments and actions on fluoride. [updated 2011 May 7; cited 2011 Feb. Available from: http://www.hhs.gov/news/press/2011pres/01/20110107a.html

[9] Head Start Bureau. [cited 2011 Aug]. Available from: http://www.acf.hhs.gov/programs/ohs

[10] Florida Medicaid. Welcome to Medicaid. [updated 2011; cited 2011 Aug. Available from: http://www.fdhc.state.fl.us/medicaid/ .

[11] Pender NJ, Murdaugh CL, Parsons MA. Health promotion in nursing practice. 5th ed. NJ: Pearson Prentice Hall; 2006.367.

[12] Sabbahi DA, Lawrence HP, Limeback H, Rootman I. Development and evaluation of an oral health literacy instrument for adults. Comm Den and Oral Epi. 2009; 37: 451-62. PMid:19740249http://dx.doi.org/10.1111/j.1600-0528.2009.00490.x

[13] Lawrence H, Romanetz R, Cappel L, Binguis D, Rogers J. Effects of a community-based prenatal nutrition program on the oral health of Aboriginal preschool children in northern Ontario. Probe. 2004; 38: 172-90.

[14] Schroth RJ, Brothwell DJ, Moffatt MEK. Caregiver knowledge and attitudes of preschool oral health and early childhood caries (ECC). Int J of Circumpolar Hlth. 2007; 66(2): 153-67. PMid:17515255http://dx.doi.org/10.3402/ijch.v66i2.18247

[15] IBM® SPSS ${ }^{\circledR}$ (2011). Statistics Standard GradPack, version 19.0.

[16] Adair PM, Pine CM, Burnside G, Nicoll AD, Gillett A, Anwar S, et al. Familial and cultural perceptions and beliefs of oral hygiene and dietary practices among ethnically and socio-economically diverse groups. Comm Den Hlth. 2004; 21(Supp): 102-11.

[17] Huskinson W, Lloyd H. Oral health in hospitalized patients: Assessment and hygiene. Nurs Standard. 2009; $23(36)$ : 43-7. PMid:19514205

[18] Melvin CS. A collaborative community-based oral care program for school-aged children. Clin Nur Spec. 2006 ; $20(1)$ : 18-22. http://dx.doi.org/10.1097/00002800-200601000-00007

[19] Miller (2011a), Adapted version from Pender's Health Promotion Model, related to oral health care.

[20] DHHS. (Department of Health and Human Services). Healthy People 2020. DC: U.S. Government Printing Services; $2000 c .15$. 